\title{
Effect of Inorganic Fertilizer on the Microbial degradation of Diesel Polluted Soil in Abeokuta, Nigeria
}

\section{*11OBUOTOR, TM; AKANDE, OA; BADA, BS. ${ }^{2}$}

\author{
${ }^{l}$ Department of Microbiology, \\ Federal University of Agriculture, Abeokuta, Ogun State, Nigeria, 110001. \\ ${ }^{2}$ Department of Environmental Management and Toxicology, \\ Federal University of Agriculture, Abeokuta, Ogun State, Nigeria, 110001. \\ *Corresponding author: Telephone number: +2348037250964 \\ E-mail: badabs@funaab.edu.ng
}

\begin{abstract}
The effect of Inorganic Fertilizer (IF) on the microbial degradation of diesel polluted soil in Abeokuta was assessed by collecting Top soil $(0-15 \mathrm{~cm}$ depth) from diesel polluted site of Information and Communication Centre, Federal University of Agriculture, Abeokuta, Nigeria. Inorganic fertilizer was added to the polluted soil in the ratio: 0:1 (control), 1:1, 2:1 and 3:1. Microorganism identification, Soil Organic Carbon (SOC) and Total Petroleum Hydrocarbon (TPH) were determined using standard methods. Data obtained were subjected to descriptive and inferential statistics. Microorganism identified were Pseudomonas aeruginosa, Proteus vulgaris, Enterobacter sp., Bacillus sp., Klebsiella sp., Saccharomyces sp., Candida sp., Fusarium sp., Trichoderma sp., Neurospora sp., Penicillium sp., Alternaria sp., Aspergilus niger, Aspergilus flavus, and Aspergilus oryzaeas. $\mathrm{pH}$ of the soil ranged between 5.8 and 7.8. Electrical conductivity, $\mathrm{NO}_{3}, \mathrm{Na}, \mathrm{Mg}$ and $\mathrm{Ca}$ contents decreased with inorganic fertilizer application. Organic Carbon, $\mathrm{PO}_{4}$ and $\mathrm{K}$ increased with fertilizer application. TPH degradation observed at 1:1, 2:1 and 3:1 were $13.38 \%, 6.45 \%$ and $10.62 \%$ respectively. (C) JASEM
\end{abstract}

http://dx.doi.org/10.4314/jasem.v19i4.4

\section{Introduction}

Petroleum products are the major source of energy for industry and daily life. Leaks and accidental spills occur regularly during the exploration, production, refining, transport, and storage of petroleum and petroleum products. Release of hydrocarbons into the environment whether accidentally or due to human activities is a main cause of water and soil pollution (Holliger et al., 1997). Soil contamination with hydrocarbons causes extensive damage of local system since accumulation of pollutants in animals and plant tissue may cause death or mutations (Alvarez and Vogel, 1991). The technology commonly used for the soil remediation includes mechanical, burying, evaporation, dispersion, and washing. However, these technologies are expensive and can lead to incomplete decomposition of contaminants while bioremediation (use of biological materials) technology is believed to be noninvasive and relatively cost-effective (April et al., 2000). Bioremediation can occur on its own (natural attenuation or intrinsic bioremediation) or can be spurred on via the addition of fertilizers to increase the bioavailability within the medium (biostimulation) or the addition of microorganisms. It has been reported that the addition of nutrients has a beneficiary effect on hydrocarbon degradation in the soil by enhancing microbial activity in organic compounds metabolization (Chaineau, 2003). Inorganic fertilizers have been used to improve soil fertility and enhance crop growth in crude oil polluted soil (Ogbogholo et al., 2005; Ijah et al., 2008). Hence this study determined the bioremediative ability of different ratio of inorganic fertilizer (N. P. K.: 15: 15:15) on diesel polluted soil collected from generator site of Information and Communication Technology Research Centre, Federal University of Agriculture, Abeokuta, Nigeria.

\section{MATERIALS AND METHODS}

Diesel polluted soil $(0-15 \mathrm{~cm}$ depth $)$ was collected from the generator site of Information and Communication Technology Research Centre, Federal University of Agriculture, Abeokuta, Nigeria.

Two litre plastic pots were filled with $1 \mathrm{~kg}$ diesel polluted soil. Inorganic fertilizer (N. P. K.: 15: 15:15) was then applied in the ratios: $1: 1,2: 1$ and $3: 1$ of inorganic fertilizer: soil. A separate pot was used for natural attenuation to serve as control. All the treatments were replicated three times. 
They were regularly turned over to allow for mixing and aeration and also watered with sterile distilled water every other day. Samples were collected every week for six weeks and stored at $4{ }^{\circ} \mathrm{c}$ for laboratory analysis. Microbial and chemical properties were analysed using standard methods.

Biodegradation efficiency was calculated using Michaud et al. (2004).

$$
\mathrm{E}=100-\frac{(\text { As } \times 100)}{\text { Aac }}
$$

As $=$ Total area of peaks in each sample,

Aac $=$ Total area of peaks in the appropriate abiotic control

$\mathrm{BE}(\%)=$ Biodegradation efficiency.

Data were subjected to descriptive and inferential statistics. Means were separated using Duncan Multiple Range Test

\section{RESULTS AND DISCUSSION}

Pseudomonas aeruginosa, Proteus vulgaris, Enterobacter sp., Bacillus sp. and Klebsiella sp.were bacteria identified (Table 1). Adebusoye et al. (2007) identified nine bacterial strains, namely, Pseudomonas fluorescens, P. aeruginosa, Bacillus subtilis, Bacillus sp., Alcaligenes sp., Acinetobacter lwoffi, Flavobacterium sp., Micrococcus roseus, and Corynebacterium sp. in petroleum hydrocarbons polluted soil in Lagos, Nigeria. Fungi identified were Saccharomyces sp. Candida sp. Fusarium sp., Trichoderma sp., Neurospora sp., Penicillium sp., Alternaria sp., Aspergilusniger, Aspergilusflavus, and Aspergilusoryzaeas (Table 2). However, Pseudomonas fluorescens, P. aeruginosa, Bacillus subtilis, Bacillus sp., Alcaligenes sp., Acinetobacter lwoffi, Flavobacterium sp., Micrococcus roseus and Corynebacterium $s p$. were isolated from polluted soil and stream (Roy et al., 2002; Okerentugba and Ezeronye, 2003; Idise et al., 2010). It is also in line with the results obtained by Ugochukwu et al. (2008) which revealed Bacillus, Fusarium, Pseudomonas, Candida and Aspergilusniger as organisms indigenous to soil that have the potential to degrade crude oil.

It was observed that fertilizer application affected the chemical properties of the soil. $\mathrm{pH}$ of the soil ranged between 5.8 and 7.8 (Table 3). $\mathrm{pH}$ of the soil increase during the study period which is in line with Das and Chandran (2011). Electrical conductivity, $\mathrm{NO}_{3}, \mathrm{Na}$, $\mathrm{Mg}$ and $\mathrm{Ca}$ contents decreased with inorganic fertilizer application (Table 4). Organic Carbon, $\mathrm{PO}_{4}$ and $\mathrm{K}$ increased with fertilizer application (Table 4). Conductivity decrease compared to the initial value and that of the control which implies a reduction in the ability of the soil to transport anions and cations.

Highest degradation was observed in $3: 1$ with percentage degradation of $11 \%$ followed by $2: 1$ with percentage degradation of $7 \%, 1: 1$ with percentage degradation of $6 \%$ and control with percentage degradation of $3 \%$ (Table 5). This might be due to the effectiveness of inorganic fertilizer (N.P.K: $15: 15: 15)$ to provide nutrients for microbial growth.

Conclusion: NPK fertilizer improved soil fertility and also enhanced degradation of Total Petroleum Hydrocarbon in diesel polluted soil.

Table 1: Oil degrading bacteria isolated from polluted soil under different levels of inorganic fertilizer

\begin{tabular}{|c|c|c|c|c|}
\hline Week & $1: 1$ & $2: 1$ & $3: 1$ & Control \\
\hline 1 & $\begin{array}{l}\text { Pseudomonas } \\
\text { aeruginosa, Enterobacter } \\
\text { specie, }\end{array}$ & $\mathrm{Ng}$ & $\begin{array}{l}\text { Pseudomonas } \\
\text { aeruginosa, }\end{array}$ & $\begin{array}{l}\text { Bacillus specie, } \\
\text { Pseudomonas aeruginosa, } \\
\text { Proteus vulgaris }\end{array}$ \\
\hline 2 & Enterobacter specie & $\begin{array}{l}\text { Pseudomonas } \\
\text { aeruginosa, Enterobacter } \\
\text { specie }\end{array}$ & $\begin{array}{l}\text { Bacillus specie, } \\
\text { Pseudomonas } \\
\text { aeruginosa, Enterobacter } \\
\text { specie }\end{array}$ & $\begin{array}{l}\text { Bacillus specie, } \\
\text { Pseudomonas aeruginosa, } \\
\text { Proteus vulgaris }\end{array}$ \\
\hline 3 & $\mathrm{Ng}$ & $\mathrm{Ng}$ & $\mathrm{Ng}$ & $\begin{array}{l}\text { Pseudomonas aeruginosa, } \\
\text { Proteus vulgaris, } \\
\text { Klebsiella specie }\end{array}$ \\
\hline 4 & $\begin{array}{lr}\text { Bacillus } & \text { specie, } \text { Proteus } \\
\text { vulgaris, } & \text { Klebsiella } \\
\text { specie } & \end{array}$ & $\begin{array}{l}\text { Pseudomonas } \\
\text { aeruginosa, } \\
\text { vulgaris, } \\
\text { specie }\end{array}$ & $\begin{array}{l}\text { Bacillus } \\
\text { Pseudomonas } \\
\text { aeruginosa, } \\
\text { vulgaris, } \\
\text { specie }\end{array}$ & $\begin{array}{l}\text { Pseudomonas aeruginosa, } \\
\text { Klebsiella specie, } \\
\text { Enterobacter specie }\end{array}$ \\
\hline 5 & $\begin{array}{l}\text { Proteus vulgaris, } \\
\text { Pseudomonas aeruginosa }\end{array}$ & $\begin{array}{l}\text { Bacillus } \\
\text { Pseudomonas } \\
\text { aeruginosa, } \\
\text { vulgaris }\end{array}$ & $\begin{array}{l}\text { Bacillus } \\
\text { Pseudomonas } \\
\text { aeruginosa, } \\
\text { vulgaris }\end{array}$ & $\begin{array}{l}\text { Bacillus specie, } \\
\text { Pseudomonas aeruginosa, } \\
\text { Proteus vulgaris, }\end{array}$ \\
\hline 6 & $\begin{array}{l}\text { Bacillus specie, } \\
\text { Pseudomonas aeruginosa }\end{array}$ & $\begin{array}{l}\text { Bacillus specie, } \\
\text { Pseudomonas aeruginosa }\end{array}$ & $\begin{array}{l}\text { Bacillus specie, } \\
\text { Pseudomonas aeruginosa }\end{array}$ & $\begin{array}{l}\text { Bacillus specie, } \\
\text { Enterobacter specie }\end{array}$ \\
\hline
\end{tabular}

Ng: No growth 
Table 2: Fungi isolated from polluted soil under different levels of inorganic fertilizer

\begin{tabular}{|c|c|c|c|c|}
\hline Week & $1: 1$ & $2: 1$ & $3: 1$ & Control \\
\hline 1 & $\begin{array}{l}\text { A. oryzae, A. flavus, } \\
\text { Saccharomyces specie }\end{array}$ & $\begin{array}{lr}\text { Candida } & \text { specie, } \\
\text { Saccharomyces } & \text { specie, A. } \\
\text { niger, A. oryzae }\end{array}$ & $\begin{array}{l}\text { Saccharomyces specie, A. } \\
\text { oryzae, Candida specie }\end{array}$ & $\begin{array}{l}\text { Saccharomyces specie, } A . \\
\text { flavus, A. niger, A. oryzae, } \\
\text { Candida specie }\end{array}$ \\
\hline 2 & $\begin{array}{l}\text { A. niger, A. oryzae, Candida } \\
\text { specie, }\end{array}$ & $\begin{array}{l}\text { Saccharomyces specie, A. } \\
\text { niger A flavus, A. oryzae }\end{array}$ & $\begin{array}{l}\text { Saccharomyces specie, A. } \\
\text { flavus, Candida specie }\end{array}$ & $\begin{array}{l}\text { Saccharomyces specie, A. } \\
\text { niger, A. flavus, Candida } \\
\text { specie }\end{array}$ \\
\hline 3 & $\begin{array}{ll}\text { Alternaria } & \text { specie, } \\
\text { Penicillium } & \text { specie, } \\
\text { Trichoderma specie } & \end{array}$ & $\begin{array}{l}\text { Trichoderma specie } A . \\
\text { niger, Penicillium specie }\end{array}$ & $\begin{array}{ll}\text { Alternaria } & \text { specie, } \quad A . \\
\text { niger, } & \text { Trichoderma } \\
\text { specie } & \end{array}$ & $\begin{array}{l}\text { A. flavus, A. } \begin{array}{r}\text { niger, } \\
\text { Penicillium } \\
\text { Trichoderma }\end{array} \text { specie, } \text { Candida } \\
\text { specie }\end{array}$ \\
\hline 4 & $\begin{array}{l}\text { Neurospora specie, } \\
\text { Penicillium specie, A. flavus }\end{array}$ & $\begin{array}{lc}\text { Neurospora } & \text { specie, } \quad \text { A. } \\
\text { flavus, } & \text { Trichoderma } \\
\text { specie, Fusarium specie }\end{array}$ & $\begin{array}{l}\text { Neurospora specie, } A . \\
\text { niger, Penicillium specie, }\end{array}$ & $\begin{array}{l}\text { Neurospora specie, } \\
\text { Penicillium specie, Fusarium } \\
\text { specie, Trichoderma specie } \\
\text { A. niger }\end{array}$ \\
\hline 5 & $\begin{array}{l}\text { Neurospora specie, Candida } \\
\text { specie, Fusarium specie }\end{array}$ & $\begin{array}{l}\text { Neurospora } \\
\text { Fusarium } \\
\text { Trichoderma specie }\end{array}$ & $\begin{array}{l}\text { Neurosporarierie, } \\
\text { Fusarium specie, A. niger }\end{array}$ & $\begin{array}{l}\text { Neurospora specie, Candida } \\
\text { specie, Fusarium specie, } \\
\text { Alternaria specie }\end{array}$ \\
\hline 6 & $\mathrm{Ng}$ & $\mathrm{Ng}$ & Saccharomyces specie & $\begin{array}{l}\text { Candida specie, Fusarium } \\
\text { specie, Alternaria specie, } \\
\text { Saccharomyces specie }\end{array}$ \\
\hline
\end{tabular}

$\mathrm{Ng}$ : No growth

Table 3: $\mathrm{pH}$ of polluted soil under different levels of inorganic fertilizer

\begin{tabular}{|c|c|c|c|c|c|c|}
\hline \multirow{2}{*}{$\begin{array}{l}\text { Fertilizer: } \\
\text { Soil }\end{array}$} & \multicolumn{3}{|c|}{ Week } & & & \multirow[t]{2}{*}{6} \\
\hline & 1 & 2 & 3 & 4 & 5 & \\
\hline $1: 1$ & 7.8 & 7.6 & 7.6 & 7.7 & 7.6 & 7.4 \\
\hline $2: 1$ & 7.7 & 7.4 & 7.4 & 7.5 & 7.3 & 7.3 \\
\hline $3: 1$ & 7.7 & 7.4 & 7.5 & 7.4 & 7.4 & 7.6 \\
\hline Control & 5.8 & 6.2 & 6.3 & 6.2 & 6.5 & 6.5 \\
\hline
\end{tabular}

Table 4: Influence of inorganic fertilizer application on soil chemical properties

\begin{tabular}{lllllllll}
\hline $\begin{array}{l}\text { Fertilizer : } \\
\text { Soil }\end{array}$ & $\mathrm{EC}\left(\mu \mathrm{s} \mathrm{cm}^{-3}\right)$ & $\mathrm{OC}(\%)$ & $\mathrm{NO}_{3}\left(\mathrm{mg} \mathrm{l}^{-1}\right)$ & $\mathrm{PO}_{4}\left(\mathrm{mg} \mathrm{l}^{-1}\right)$ & $\mathrm{K}\left(\mathrm{mg} \mathrm{l}^{-1}\right)$ & $\mathrm{Na}\left(\mathrm{mg} \mathrm{kg}^{-1}\right)$ & $\mathrm{Mg}\left(\mathrm{mg} \mathrm{kg}^{-1}\right)$ & $\mathrm{Ca}_{\left(\mathrm{mg} \mathrm{kg}^{-1}\right)}$ \\
\hline Initial & 2.19 & 13.60 & 32.08 & 27.12 & 0.45 & 0.50 & 9.65 & 2.55 \\
Control & 2.10 & 13.80 & 48.02 & 20.21 & 0.48 & 0.35 & 3.40 & 2.15 \\
$1: 1$ & 1.21 & 14.00 & 22.30 & 33.43 & 3.01 & 0.43 & 6.99 & 2.35 \\
$2: 1$ & 1.62 & 14.10 & 21.06 & 31.08 & 3.19 & 0.45 & 7.15 & 2.45 \\
$3: 1$ & 1.58 & 14.20 & 23.04 & 32.17 & 3.21 & 0.44 & 7.14 & 2.47 \\
\hline
\end{tabular}

Table 5: Effect of inorganic fertilizer on the degradation of

Total Petroleum Hydrocarbon.

\begin{tabular}{lll}
\hline $\begin{array}{l}\text { Fertilizer : } \\
\text { Soil }\end{array}$ & $\begin{array}{l}\text { Total Petroleum } \\
\text { Hydrocarbon } \\
\left(\mathrm{g} \mathrm{kg}^{-1}\right)\end{array}$ & $\begin{array}{l}\text { Percentage } \\
\text { Degradation }(\%)\end{array}$ \\
\hline Control & $86.03^{\mathrm{a}}$ & 3.38 \\
$1: 1$ & $83.29^{\mathrm{ab}}$ & 6.45 \\
$2: 1$ & $82.86^{\mathrm{ab}}$ & 6.94 \\
$3: 1$ & $79.58^{\mathrm{b}}$ & 10.62 \\
\hline
\end{tabular}

Means in the same column followed by the same superscript are not significantly $(\mathrm{p}<0.05)$ different (DMRT)

\section{REFERENCES}

Adebusoye, S A, Ilori, M O, Amund, O O, Teniola, O D; Olatope, S O (2007). Microbial degradation of petroleum hydrocarbons in a polluted tropical stream. World $\mathbf{J}$ of Microbio and Biotech 23(8):1149 - 1159
April, T M, Foght, J M; Currah, R S (2000). Hydrocarbon-degrading filamentous fungi isolated from flare pit soils in northern and western Canada. Canadian $\mathbf{J}$ of Microbio. 46(1):38-49 
Alvarez, P J J; Vogel, T M (1991). Substrate interactions of benzene, toluene and para-xylene during microbial degradation by pur cultures and mixed culture aquifer slurries. Applied and Enviro Microbio 57: 2981 - 2985

Chaineau, C H, Yepremian, C, Vidalie, J F, Ducreux, J; Ballerini, D (2003). Bioremediation of a crude oil-polluted soil: biodegradation, leaching and toxicity assessments. Water, Air and Soil Pollu $44: 419-440$

Das, N; Chandran, P (2011). Microbial Degradation of Petroleum Hydrocarbon Contaminants: An Overview. Biotech Res Inter 2 - 13.

Holliger, C, Gaspard, S, Glod, G, Heijman, C, Schumacher, W, Schwarzenbach, R P; Vazquez, $F$ (1997). Contaminated environment in the subsurface and bioremediation: Organic contaminants. FEMS Microbio Rev 20(3-4): 517 -523 .

Idise, O E, Ameh, J B, Yakubu, S E; Okuofu, C A (2010). Modification of Bacillus cereus and Pseudomonas aeruginosa isolated from a petroleum refining effluent for increased petroleum product degradation. African $\mathrm{J}$ of Biotech 9(22): 3303 - 3307

Ijah, U J J, Safiyanu, H; Abioye, O P (2008). Comparative study of Biodegradation of Crude oil in soil amended with Chicken droppings and NPK fertilizer. Sci World J 3(2): 63 - 67
Michaud, L, Lo Giudice, A, Saitta, M; De DomenicoVivia, M (2004). The biodegradation month-long experiment. Mar Res Bull 49: 405 409

Ogboghodo, I A, Azenabor, U F; Osemwota, I O (2005). Amelioration of Crude oil polluted soil with poultry manure and the effect on growth of maize and some soil properties. J of Plant Nut 28(1): $21-32$

Okerentugba, P O; Ezeronye, O U (2003). Petroleum degrading potentials of single and mixed microbial cultures isolated from rivers and effluents in Nigeria. Afr J Biotech 2(9): 288 292

Roy, S, Hens, D, Biswas, D; Kumar, R (2002).Survey of petroleumdegrading bacteria in coastal waters of Sunderban Biosphere Reserve. World $\mathrm{J}$ of Microbio and Biotech 18: 575 - 581

Ugochukwu, N H, Babady, N E, Cobourne, M; Gasset, S R (2003). The effect of Gangronema latifolium extracts on serum lipid profile and oxidative stress in hepatocytes of diabetic rats. J Biosci 28(1): 1 - 5 